\title{
Erratum to: GLP-1: benefits beyond pancreas
}

\author{
G. Muscogiuri - A. Cignarelli $\cdot$ F. Giorgino $~ F$. Prodam • \\ D. Santi - G. Tirabassi - G. Balercia - R. Modica ·

\section{A. Faggiano $\cdot$ A. Colao}

Published online: 2 November 2014

(C) Italian Society of Endocrinology (SIE) 2014

\section{Erratum to: J Endocrinol Invest}

\section{DOI: 10.1007/s40618-014-0137-y}

In the original publication of the article, the family name of the fourth author was wrongly spelled as F. Prodram. It should be replaced by F. Prodam.

The name should also be corrected in the affiliation details as follows:

The online version of the original article can be found under doi:10.1007/s40618-014-0137-y.

G. Muscogiuri $(\bowtie) \cdot$ R. Modica $\cdot$ A. Faggiano · A. Colao Section of Endocrinology, Department of Clinical Medicine and Surgery, University "Federico II", Via Sergio Pansini, 5, Naples, Italy

e-mail: giovanna.muscogiuri@gmail.com

\section{A. Cignarelli $\cdot$ F. Giorgino}

Section of Internal Medicine, Endocrinology, Andrology and

Metabolic Diseases, Department of Emergency and Organ

Transplantation, University of Bari Aldo Moro, Bari, Italy

\section{F. Prodam}

Division of Pediatrics, Department of Health Sciences,

University of Piemonte Orientale "A. Avogadro", Alessandria,

Italy

D. Santi

Section of Endocrinology, Department of Biomedical, Metabolic and Neural Sciences, University of Modena and Reggio Emilia, Via Campi 287, 41125 Modena, Italy

G. Tirabassi · G. Balercia

Division of Endocrinology, Department of Clinical and Molecular Sciences, Umberto I Hospital, Polytechnic

University of Marche, Ancona, Italy

\section{F. Prodam}

Division of Pediatrics, Department of Health Sciences, University of Piemonte Orientale “A. Avogadro", Alessandria, Italy 